\title{
Evaluation of Aortic Stiffness (Aortic Pulse-Wave Velocity) Before and After Elective Abdominal Aortic Aneurysm Repair Procedures: A Pilot Study ${ }^{\#}$
}

Kosmas I. Paraskevas ${ }^{1, *}$ Nikolaos Bessias ${ }^{1}$, Chrysovalantis Psathas ${ }^{2}$, Konstantinos Akridas ${ }^{2}$, Theodoros Dragios ${ }^{2}$, Georgios Nikitas ${ }^{2}$, Vassilios Andrikopoulos ${ }^{1}$,Dimitri P. Mikhailidis ${ }^{3}$ and Zenon S. Kyriakides ${ }^{2}$

\author{
${ }^{I}$ Department of Vascular Surgery and ${ }^{2}$ Department of Cardiology, Red Cross Hospital, Athens, Greece \\ ${ }^{3}$ Department of Clinical Biochemistry (Vascular Disease Prevention Clinics), Royal Free Hospital Campus, University \\ College London Medical School, University College London (UCL), London, UK
}

\begin{abstract}
Background: The main clinical criterion for abdominal aortic aneurysm (AAA) repair operations is an AAA diameter $\geq 5.5 \mathrm{~cm}$. When AAAs increase in size, specific changes occur in the mechanical properties of the aortic wall. Pulse-wave velocity (PWV) has been used as an indicator of vascular stiffness. A low PWV may predict AAA rupture risk and is an early predictor of cardiovascular mortality.

Methods: We investigated the prognostic value of PWV before and after elective AAA repair procedures. Twenty four patients scheduled for an open AAA repair underwent a preoperative carotid-femoral aortic PWV measurement. A second aortic PWV measurement was carried out 6 months postoperatively.

Results: The mean aortic PWV increased from $7.84 \pm 1.85$ preoperatively to $10.08 \pm 1.57 \mathrm{~m} / \mathrm{sec} 6$ months postoperatively (mean change: 2.25 ; $95 \%$ confidence interval 1.4 to $3.1 \mathrm{~m} / \mathrm{sec}$; $\mathrm{p}<0.0001$ ). The preprocedural PWV measurement did not correlate with AAA diameter (Spearman's rank correlation coefficient $\rho=0.12 ; \mathrm{p}=0.59$ ).

Conclusions: Whether the increase in aortic PWV postoperatively suggests a decreased cardiovascular risk following AAA repair remains to be established. Aortic PWV should also be investigated as an adjunct tool for assessing AAA rupture risk.
\end{abstract}

Keywords: Abdominal aortic aneurysm, pulse-wave velocity, aneurysm rupture risk, cardiovascular mortality, predictor.

\section{INTRODUCTION}

Currently the main criterion for abdominal aortic aneurysm (AAA) repair is an AAA diameter $\geq 5.5 \mathrm{~cm}[1]$. However, some AAAs rupture when they are smaller, whereas many AAAs are discovered when they have exceeded this critical diameter but have not ruptured [2,3]. It therefore seems that size alone is not a sufficient criterion to determine AAA rupture risk.

The carotid-femoral aortic pulse-wave velocity (PWV) is a simple and reproducible test for estimating arterial stiffness [4]. Aortic PWV is considered as the "gold standard" measurement of arterial stiffness [4]. The aortic PWV may predict AAA rupture risk and is a marker of cardiovascular event risk [4-6].

\footnotetext{
*Address correspondence to this author at the Department of Vascular Surgery, Red Cross Hospital, 24 Al. Papagou street, Athens 14122, Greece, E-mail: paraskevask@hotmail.com

"Presented as an Oral Presentation at the Larissa International Vascular and Endovascular Symposium (LIVES) 2009 in Larissa, Greece (May 2931, 2009): Available at: http://www.uth-vascularsymposium2009.gr/lives/ oral_pres.php
}

We evaluated the aortic PWV before and 6 months after elective open infrarenal AAA repair procedures.

\section{MATERIALS AND METHODS}

Twenty four patients (23 males, 1 female; mean age: $72 \pm$ 5 years) scheduled for elective open infrarenal AAA repair at the Department of Vascular Surgery of the Red Cross Hospital of Athens, Greece between April 1, 2008 and November 1, 2008 were included in this pilot study. All patients gave their informed consent before entering the study. The Ethics Committee of our Hospital approved the study.

Carotid-femoral aortic PWV measurement was performed by 2 clinicians who were familiar with the technique. Measurements were carried out in the morning after an overnight fast under controlled room temperature with constant noise and light intensity, using a validated non-invasive device (Complior SP, Artech Medical, Pantin, France) [7]. This allows online recording and automatic calculation of carotidfemoral aortic PWV.

In brief, all subjects rested for 15-20 min while pulse rate and blood pressure were measured at the brachial artery 
using an automatic oscillometric device (Dinamap XL, Johnson \& Johnson Inc, Raritan, New Jersey USA). Measurements of carotid-femoral aortic PWV were performed 2 days before the procedure while the patients were in the supine position with a slight extension of the head and with the right lower limb in external rotation (Fig. 1). Two different pulse wave tracings were recorded simultaneously at 2 sites (at the base of the neck for the common carotid artery and over the right femoral artery) with 2 transducers (Fig. 2). Five con- secutive measurements were performed in each patient and the mean carotid-femoral aortic PWV value was obtained.

All patients underwent open aneurysmectomy through a transperitoneal approach according to a standard, established technique [8]. A polytetrafluoroethylene aortobifurcated (aortobi-iliac or aortobi-femoral) graft was implanted in all patients. The aortic PWV measurement was repeated 6 months after the procedure.

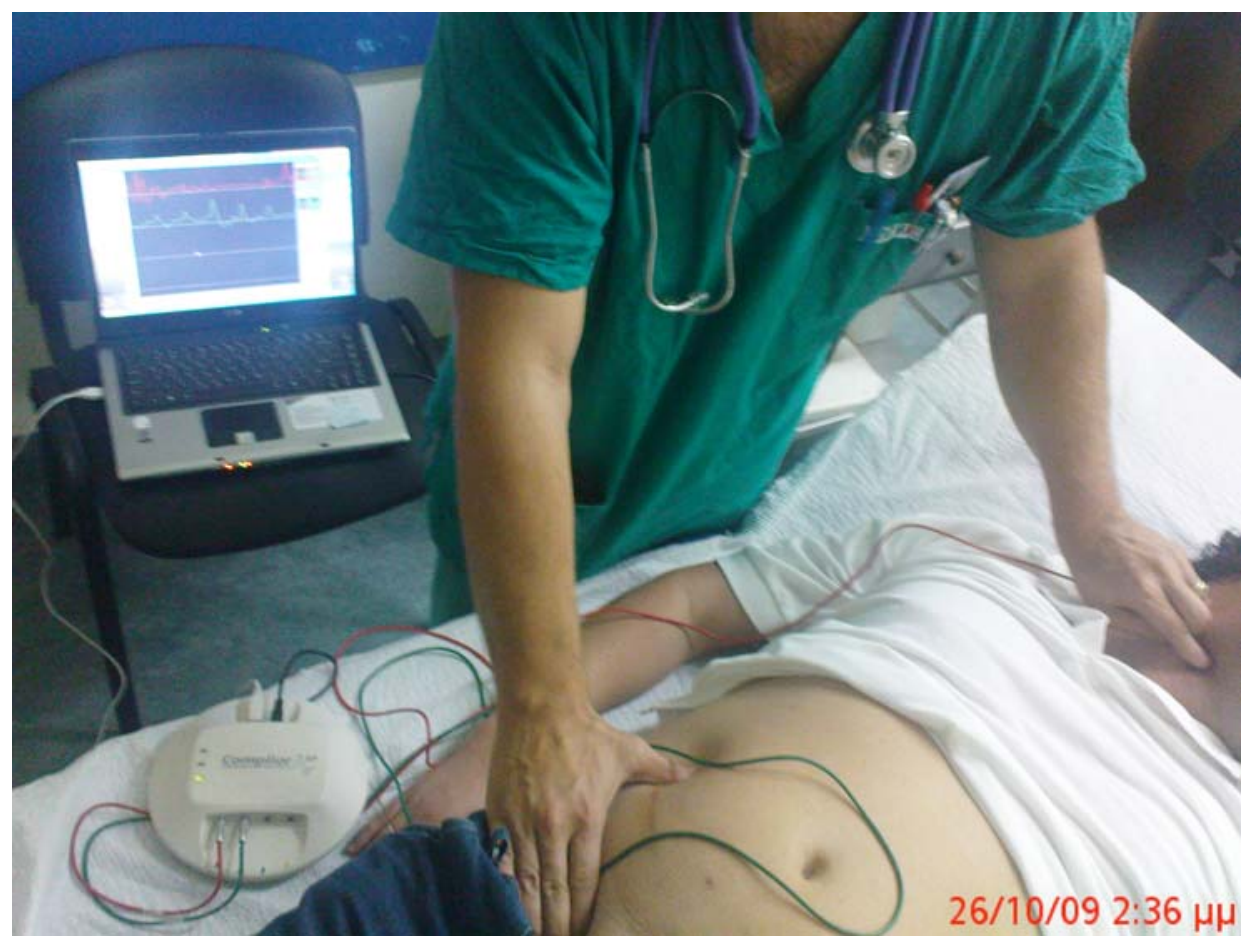

Fig. (1). Measurement of the carotid-femoral aortic pulse-wave velocity using an automatic oscillometric device (Dinamap XL, Johnson \& Johnson Inc, Raritan, New Jersey USA). For details of the technique see text.

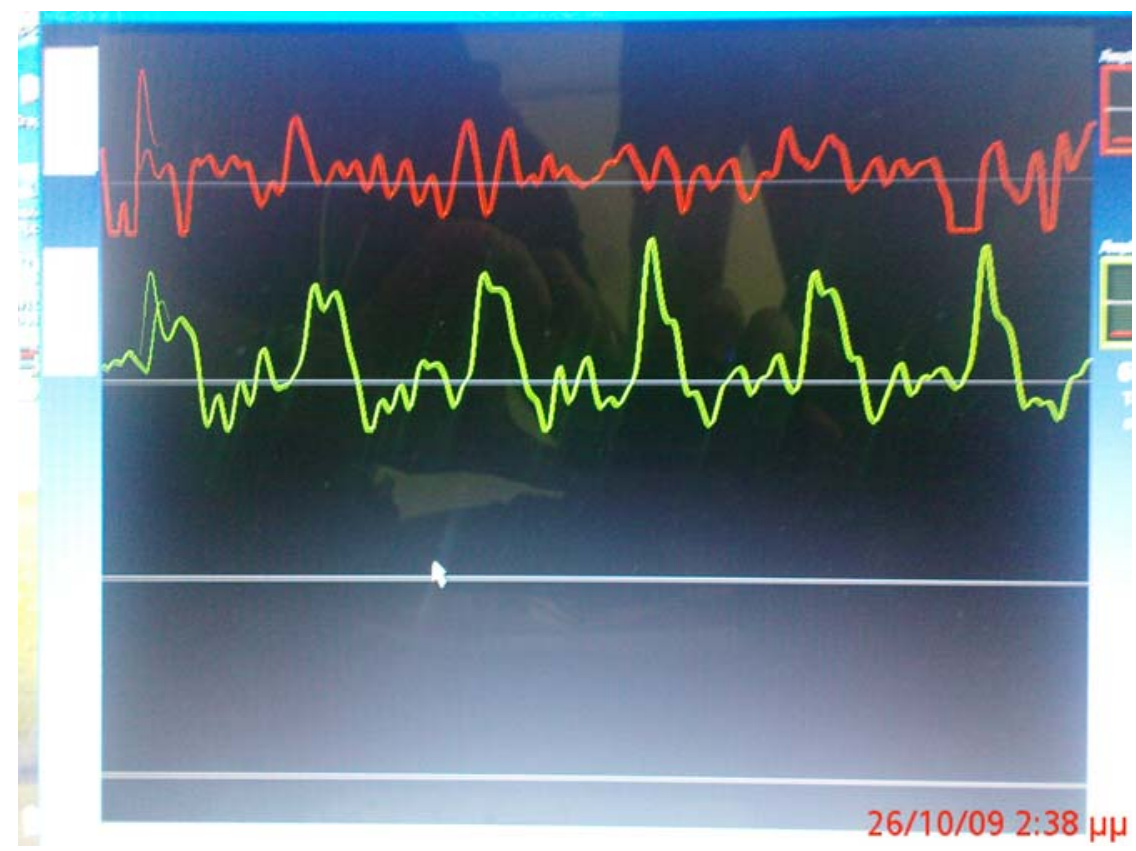

Fig. (2). Pulse-wave measurement as recorded by the oscillometric device. 


\section{STATISTICAL ANALYSIS}

The data were analyzed using SPSS 15.0 for Windows (Chicago, Illinois, USA). Continuous parameters are reported as mean \pm standard deviation or median and (range). Their distribution was evaluated by the KolmogorovSmirnov test. The t-test or the Mann-Whitney test was used to compare continuous variables, as appropriate. The Spearman's rank correlation test $(\rho)$ was performed to assess relationships between aortic PWV and AAA diameter. A p $<0.05$ was considered significant.

\section{RESULTS}

There were no perioperative deaths. Two patients suffered a minor myocardial infarction postoperatively which was treated conservatively. The mean (range) postoperative hospital stay was 7.2 (6-10) days. All patients attended a follow-up visit at 6 months postoperatively.

The mean (range) AAA diameter preoperatively was 6.3 $(5.5-8) \mathrm{cm}$. The mean aortic PWV increased from $7.84 \pm$ 1.85 preoperatively to $10.08 \pm 1.57 \mathrm{~m} / \mathrm{sec} 6$ months postoperatively (mean change: $2.25 ; 95 \%$ confidence interval: 1.4 to $3.1 \mathrm{~m} / \mathrm{sec} ; \mathrm{p}<0.0001)$.

The aortic PWV before the operation did not correlate with AAA diameter $(\rho=0.12 ; \mathrm{p}=0.594)$.

\section{DISCUSSION}

The results of our pilot study demonstrate that aortic PWV, a potential predictor of AAA rupture and cardiovascular mortality [4-6], increases following open elective AAA repair. Furthermore, aortic PWV did not correlate with AAA diameter.

The lack of correlation between the aortic PWV with AAA size in our study suggests that size alone may not be a sufficient criterion to determine AAA rupture risk. The finding that the aortic PWV increased following the procedures supports the hypothesis that a low aortic PWV is associated with AAAs at high risk of rupture [4-6].

In the normal aortas the propagation of the pulse wave is relatively uniform along the wall. AAA disease leads to mechanical changes in the aortic wall which include lower aortic distensibility and a lower propagation of the aortic pulse pressure (i.e. a lower PWV) [7]. This explains the low PWV values preoperatively. Following successful AAA repair, the velocity of the propagation of the aortic pulse wave is restored to normal. This explains the increased PWV values 6 months postoperatively. The increase in aortic PWV following AAA repair may provide a justification for an association between aortic PWV with AAA rupture risk; the high AAA rupture risk preoperatively (as demonstrated by a low aortic PWV) is eliminated following the procedure as reflected by the increase in aortic PWV.
A limitation of our study is its small sample size $(n=24)$. However, this number of patients was adequate to provide significant results. Patient enrollment is ongoing.

\section{CONCLUSIONS}

The increase in aortic PWV following open elective AAA repair suggests a decreased AAA rupture risk and cardiovascular mortality. This needs to be confirmed in larger studies. Aortic PWV may also become an adjunct tool for assessing AAA rupture risk. Endovascular AAA repair (EVAR) has emerged as a therapeutic alternative for the treatment of AAAs. Future trials should assess the value of measuring aortic PWV before and after EVAR procedures and compare these changes with open surgical AAA repair.

\section{REFERENCES}

[1] Hirsch AT, Haskal ZJ, Hertzer NR, et al. American Association for Vascular Surgery; Society for Vascular Surgery; Society for Cardiovascular Angiography and Interventions; Society for Vascular Medicine and Biology; Society of Interventional Radiology; ACC/AHA Task Force on Practice Guidelines Writing Committee to Develop Guidelines for the Management of Patients With Peripheral Arterial Disease; American Association of Cardiovascular and Pulmonary Rehabilitation; National Heart, Lung, and Blood Institute; Society for Vascular Nursing; TransAtlantic Inter-Society Consensus; Vascular Disease Foundation. ACC/AHA 2005 Practice Guidelines for the management of patients with peripheral arterial disease (lower extremity, renal, mesenteric, and abdominal aortic): a collaborative report from the American Association for Vascular Surgery/Society for Vascular Surgery, Society for Cardiovascular Angiography and Interventions, Society for Vascular Medicine and Biology, Society of Interventional Radiology, and the ACC/AHA Task Force on Practice Guidelines (Writing Committee to Develop Guidelines for the Management of Patients With Peripheral Arterial Disease): endorsed by the American Association of Cardiovascular and Pulmonary Rehabilitation; National Heart, Lung, and Blood Institute; Society for Vascular Nursing; TransAtlantic Inter-Society Consensus; and Vascular Disease Foundation. Circulation 2006; 113: e463-654.

[2] Fillinger M. Who should we operate on and how do we decide: predicting rupture risk and survival in patients with aortic aneurysm. Semin Vasc Surg 2007; 20: 121-7.

[3] Reeps C, Essler M, Pelisek J, Seidl S, Eckstein HH, Krause BJ. Increased $18 \mathrm{~F}$-fluorodeoxyglucose uptake in abdominal aortic aneurysms in positron emission/computed tomography is associated with inflammation, aortic wall instability, and acute symptoms. J Vasc Surg 2008; 48: 417-23.

[4] Laurent S, Cockcroft J, Van Bortel L, et al. European Network for Non-invasive Investigation of Large Arteries. Expert consensus document on arterial stiffness: methodological issues and clinical applications. Eur Heart J 2006; 27 : 2588-605.

[5] Luo J, Fujikura K, Tyrie LS, Tilson MD, Konofagou EE. Pulse wave imaging of normal and aneurysmal abdominal aortas in vivo. IEE Trans Med Imaging 2009; 28: 477-86.

[6] Meaume S, Benetos A, Henry OF, Rudnichi A, Safar ME. Aortic pulse wave velocity predicts cardiovascular mortality in subjects $>70$ years of age. Arterioscler Thromb Vasc Biol 2001; 21; 2046-50.

[7] Fujikura K, Luo J, Gamarnik V, et al. A novel noninvasive technique for pulse-wave imaging and characterization of clinicallysignificant vascular mechanical properties in vivo. Ultrason Imaging 2007; 29: 137-54.

[8] Carroccio A, Hollier LH. Abdominal aortic aneurysm. Haimovici's Vascular Surgery $5^{\text {th }}$ Edition. Blackwell Publishing Inc., 2004; pp. 703-35. 